\title{
Field theory on multithroat backgrounds
}

\author{
Giacomo Cacciapaglia, ${ }^{1, *}$ Csaba Csáki, ${ }^{1, \dagger}$ Christophe Grojean, ${ }^{2,3, \ddagger}$ and John Terning ${ }^{4, \S}$ \\ ${ }^{1}$ Institute for High Energy Phenomenology Newman Laboratory of Elementary Particle Physics, \\ Cornell University, Ithaca, New York 14853, USA \\ ${ }^{2}$ CERN Physics Department, Theory Division, CH-1211 Geneva 23, Switzerland \\ ${ }^{3}$ Service de Physique Théorique, CEA Saclay, F91191 Gif-sur-Yvette, France \\ ${ }^{4}$ Department of Physics, University of California, Davis, California 95616, USA
}

(Received 4 July 2006; published 18 August 2006)

\begin{abstract}
We consider extra dimensional field theory descriptions of backgrounds with $N$ different throats where one of the extra dimensions in each throat is much larger than the others. Such backgrounds can be described by field theory on $N$ 5D warped spaces which intersect on a ultraviolet (UV) brane. Given a field that propagates in all $N$ throats there are $N$ boundary conditions on the UV brane (which are determined by the effective Lagrangian on the UV brane) in addition to the boundary conditions on the $N$ infrared branes. We derive a general set of UV boundary conditions and give examples of how they are applied to particular situations. Three simple example applications are given: in the first the number of families is determined by the number of throats and the SUSY flavor problem is solved via an $S_{3}$ symmetry of the throats; in the second we embed this scenario in a SUSY GUT with a solution of the doublet-triplet splitting problem based on the product group approach; while in the final example we show a simple geometric implementation of a SUSY trinification model on three throats.
\end{abstract}

DOI: 10.1103/PhysRevD.74.045019

PACS numbers: 12.60.-i, 11.10.Kk, 11.25.Wx, 12.60.Cn

\section{INTRODUCTION}

Theories with extra dimensions have found many new applications over the past ten years. Large and warped extra dimensions have been proposed to alleviate the hierarchy problem [1,2], small extra dimensions can give rise to novel approaches to GUT model building [3], universal extra dimensions can give new dark matter candidates [4], and even new mechanisms for electroweak symmetry breaking can be found using extra dimensions [5-7]. In most of these models, only the simplest possible topologies of the extra dimensional space have been assumed: a single (or sometimes two) extra dimensions compactified on a circle (or torus), or a very simple orbifold like $S^{1} / Z_{2}$ or $T^{2} / Z_{2}$. This severely limits the possible configurations available for model building. For example in the case of warped extra dimensions it is usually assumed that there is a single warp factor in the theory, and that one needs to introduce complicated intersecting brane configurations in order to get a warped model in more than one extra dimension. However, it has been found in string theory [8] that a generic type of situation arising from flux compactifications leads to geometries with multiple "throats" hanging out from the "head", which is a compact CalabiYau manifold (see Fig. 1). Such compactifications with multiple throats could have many advantages over the more commonly considered ones. It is relatively simple to introduce multiple scales into the theory, it allows for

\footnotetext{
*Electronic address: cacciapa@mail.lns.cornell.edu

${ }^{\dagger}$ Electronic address: csaki@lepp.cornell.edu

Electronic address: grojean@spht.saclay.cea.fr

${ }^{\S}$ Electronic address: terning@physics.ucdavis.edu
}

clearly separate fields without having to introduce multiple extra dimensions, etc. The purpose of this paper is to give a simple field theory description of such multithroat string compactification set-ups, and to show some possible simple applications for extra dimensional model building that would not be easily achieved without such set-ups (see also [9] for previous phenomenological applications of multithroat set-ups).

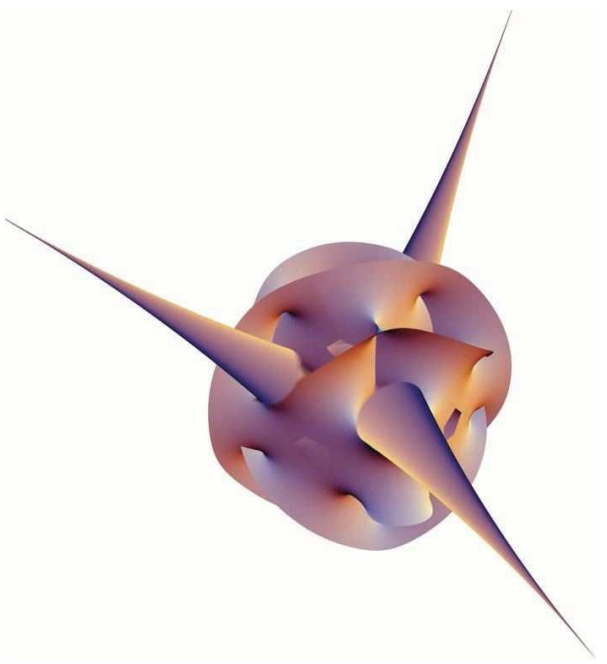

FIG. 1 (color online). A generic multithroat geometry. Multiple throats are hanging out from the head which is a compact Calabi-Yau manifold. In our field theory approach, each throat is modeled by a single warped extra dimension. The throats intersect at a point, the UV brane. The UV physics of the head is described by some brane localized operators connecting the various fields living in the individual throats. 


\section{BOUNDARY CONDITIONS}

In the field theory limit that we are considering we imagine that the transverse sizes of the throats are all negligible, and also shrink the head to a single point, the UV brane. Thus we end up with $N$ 5D bulks parametrized by the coordinates $z_{1}, \ldots, z_{N}$ (see Fig. 2). We should again stress that these are not coordinates of $N$ extra dimensions, but rather $N$ single extra dimensions intersecting at a point in space, thus identifying the points ${ }^{1} z_{1}=0$ with $z_{2}=$ $0, \ldots, z_{N}=0$. This point is what we will be calling the UV brane. The other $N$ (distinct) endpoints of the throats corresponding to $z_{1}=R_{1}^{\prime}, z_{2}=R_{2}^{\prime}, \ldots, z_{N}=R_{N}^{\prime}$ will correspond to $N$ separate IR branes, thus yielding $N$ separate scales in the theory. This set-up is a generalization to multithroats of our construction [10] where two throats have been introduced to separate the top and bottom quarks from the light fermions.

On this geometry, let us consider a single scalar field $\phi$ which propagates in all of the throats. We start our analysis of the modes of this field by introducing $N$ 5D bulk scalar fields $\phi_{i}$ each of which will live in just one of the throats that intersect on the single UV brane. The bulk actions are given by ${ }^{2}$

$$
\mathcal{L}_{\text {bulk }}=\sum_{i} \sqrt{g_{(i)}}\left(\frac{1}{2} g_{(i)}^{M N} \partial_{M} \phi_{i} \partial_{N} \phi_{i}-V_{i}\left(\phi_{i}\right)\right),
$$

where $g_{i}$ is the metric in the $i$-th throat. In order for the picture to be consistent, they have to match on the UV brane: $g_{(1) \mid z_{1}=\mathrm{UV}}=\ldots=g_{(N) z_{N}=\mathrm{UV}}=g_{(\mathrm{UV})}$. At this point, the $N$ fields $\phi_{i}$ are still totally independent. In order to make it a single field propagating over the whole geometry we need to connect these fields at the UV brane. For simplicity we will first consider only the lowest dimension operators on the UV brane (warp factor dependencies are included in the mass terms):

$$
\mathcal{L}_{\mathrm{UV}}=\left(-\frac{1}{2} \sum_{i, j=1}^{N} m_{i j} \phi_{i} \phi_{j}\right)_{I_{i}=\mathrm{UV}} .
$$

The variation of the bulk actions and the UV-brane action gives the following $N$ boundary conditions (BC's):

$$
0=\partial_{z_{i}}\left(g_{(i)}^{55} \sqrt{g_{(i)}} \phi_{i}\right)+\sum_{j=1}^{N} m_{i j} \phi_{j} .
$$

UV-brane kinetic terms can be included in a straightfor-

\footnotetext{
${ }^{1}$ For a flat extra dimension, we can always chose the UV brane in each throat to be located at $z_{i}=0$. In a warped extra dimension where translational invariance along the extra dimension is lost, we cannot simply shift the origin of the coordinates $z_{i}$ to bring the UV brane at $z_{i}=0$. The discussion presented here is easily generalized to more general (warped) set-ups.

${ }^{2}$ Throughout this paper, we will use a mostly minus signature and $M, N \ldots=0,1,2,3,5$ denote bulk coordinates while $\mu, \nu \ldots=0,1,2,3$ denote brane coordinates.
}

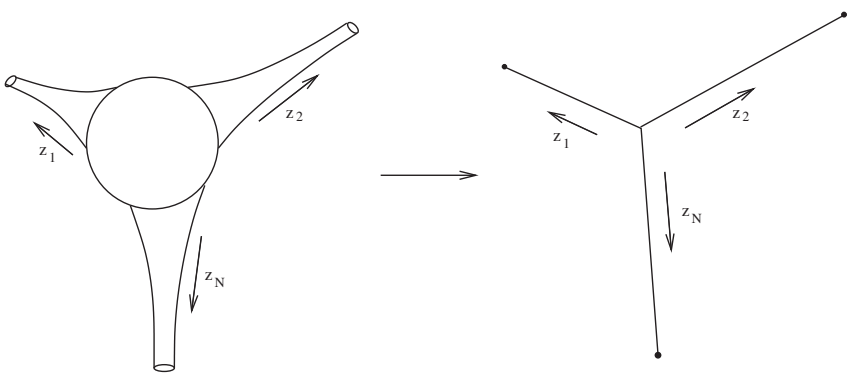

FIG. 2. Illustration of the limit of the generic multithroat geometry considered in this paper.

ward fashion, leading to $\partial^{\mu} \partial_{\mu} \phi_{i}$ terms in the BC. Including interaction terms on the UV brane will lead to nonlinear terms in the BC's. Note, that (2.3) provides exactly the right number of BC's to be able to fully determine the system. We started out with $N$ scalar fields each of which satisfy a second order differential equation, so one needs $2 N$ BC's to solve the system. $N$ of these are provided by the BC's at the IR branes, while further $N$ come from (2.3) at the UV brane.

A particular choice of mass terms on the UV brane leads to a very simple set of BC's. If the mass terms have the form (with the convention that $\phi_{N+1}=\phi_{1}$ )

$$
\mathcal{L}_{\mathrm{UV}}=-\sum_{i=1}^{N} k_{i} m\left(\phi_{i}-\phi_{i+1}\right)_{\mid z_{i}=\mathrm{UV}}^{2}
$$

then the $\mathrm{BC}$ 's are

$$
\partial_{z_{i}}\left(g_{(i)}^{55} \sqrt{g_{(i)}} \phi_{i}\right)+2 k_{i} m\left(\phi_{i}-\phi_{i+1}\right)=0 .
$$

In the large $m$ limit these equations will just simplify to the $N-1$ independent equations (independent of the $k_{i}$ 's):

$$
\phi_{i}=\phi_{i+1} \quad \text { for } i=1 \ldots N-1
$$

while the sum of these equations will give the remaining $\mathrm{BC}$

$$
0=\sum_{i=1}^{N} \frac{1}{k_{i}} \partial_{z_{i}}\left(g_{(i)}^{55} \sqrt{g_{(i)}} \phi_{i}\right)
$$

A mechanical analogy for this system is given by $N$ vibrating rods, which are connected at their ends with springs to each other, with the boundary mass $k_{i} m$ corresponding to the spring constant (see Fig. 3).

In a more general case, the structure of the spectrum, in particular, the number of zero modes and their localization in the throats, depends on the $\mathrm{BC}$ on the UV brane, effectively described by the localized mass terms. The general set of BC's in Eq. (2.3) can be rewritten as:

$$
0=\partial_{z_{i}} \phi_{i}+\sum_{j=1}^{N} \tilde{m}_{i j} \phi_{j},
$$

where 


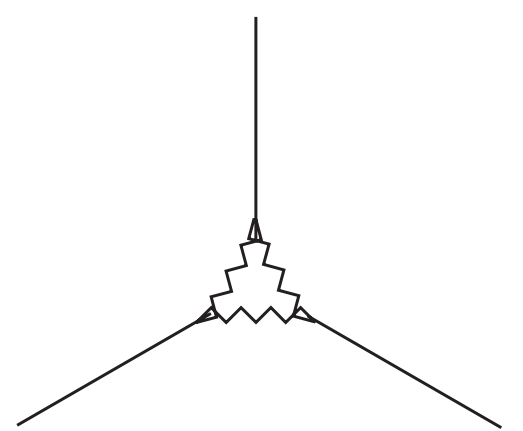

FIG. 3. A mechanical analogy: our procedure of generating the BC's on the UV brane can be thought of as vibrating rods connected by springs at one of their ends.

$$
\tilde{m}_{i j}=\left(g_{(\mathrm{UV})}^{55} \sqrt{g_{(\mathrm{UV})}}\right)^{-1}\left(\partial_{z_{i}}\left(g_{(i)}^{55} \sqrt{g_{(i)}}\right) \delta_{i j}+m_{i j}\right)_{\mid z_{i}=\mathrm{UV}}
$$

(we have used here the continuity of the metric on the UV brane, and simply redefined the mass term in order to include the derivative of the metric factor). Let us assume for simplicity that bulk mass terms are absent and the BC's on the IR branes are Neuman: in this case the zero modes are characterized by flat wave functions $\phi_{i}=$ const. Therefore, there is a zero mode for every BC involving only derivatives: after diagonalizing the mass matrix $\tilde{m}$, we can see that the number of zero modes is equal to the number of zero eigenvalues $(N$-rank $\tilde{m})$. The eigenstates also determine where the zero modes live. Take $v_{i}^{(a)}$ to be the components of $a$-th eigenvector with eigenvalue 0 (i.e. $a$ runs from 1 to $N$-rank $\tilde{m}$ ). Then the solutions for the constant zero modes are

$$
\phi_{i}^{(a)} \propto v_{i}^{(a)} .
$$

If $v_{k}$ is smaller that the other coefficients, the component of the zero mode living in the $k$-th throat is suppressed with respect to the others, so that the zero mode is repelled from the $k$-th throat. On the other hand, if $v_{k}$ is larger than the other $v$ 's, than the zero mode lives preferably in the $k$-th throat. In this way, just playing with the BC's we can control where a scalar zero mode is living. For the more general case with nonconstant zero modes bulk solutions (but still assuming that the warp factors and bulk masses are the same in each throat), the actual zero modes correspond to eigenvectors of $\tilde{m}_{i j}$ whose eigenvalues are the negative of the slope of the zero mode bulk solution. It is straightforward to generalize this to the case where the bulk zero mode solutions are different in each throat.

It is easy to check that for a flat bulk with the general UV BC's (2.3) that the standard reflection/transmission problem works correctly. We can for instance check it explicitly in the nontrivial case of three throats connected at a UV brane by general BC's of the form (2.3). Taking IR BC's such that on one bulk there is an incoming (towards the UV brane) plane wave and a reflected plane wave, while in the other bulks there are transmitted (moving away from the UV brane) plane waves, the wave functions of the three scalar fields will be of the form (the UV BC's require the waves to have the same frequency in the different throats and, assuming the same bulk mass in the different throats, the bulk equations of motion also impose the wave vectors to have the same strength)

$$
\begin{gathered}
\phi_{1}\left(x, z_{1}\right)=e^{i\left(\omega t-k z_{1}\right)}+r e^{i\left(\omega t+k z_{1}\right)} \\
\phi_{2}\left(x, z_{2}\right)=\alpha_{2} e^{i\left(\omega t+k z_{2}\right)} \\
\phi_{3}\left(x, z_{3}\right)=\alpha_{3} e^{i\left(\omega t+k z_{3}\right)}
\end{gathered}
$$

$r$ is the reflection coefficient in the first throat, while $\alpha_{2,3}$ are the transmission coefficients in the other throats. The general BC's at the UV brane $\left(z_{i}=0\right)$ are

$$
\begin{aligned}
& \partial_{z_{1}} \phi_{1 \mid z_{1}=0}+m_{11} \phi_{1 \mid z_{1}=0}+m_{12} \phi_{2 \mid z_{2}=0}+m_{13} \phi_{3 \mid z_{3}=0}=0 \\
& \partial_{z_{2}} \phi_{2 \mid z_{2}=0}+m_{12} \phi_{1 \mid z_{1}=0}+m_{22} \phi_{2 \mid z_{2}=0}+m_{23} \phi_{3 \mid z_{3}=0}=0 \\
& \partial_{z_{3}} \phi_{3 \mid z_{3}=0}+m_{13} \phi_{1 \mid z_{1}=0}+m_{23} \phi_{2 \mid z_{2}=0}+m_{33} \phi_{3 \mid z_{3}=0}=0
\end{aligned}
$$

These three equations can be solved to obtain the reflection and transmissions coefficients in terms of the UV-brane masses, $m_{i j}$. For example, in the simplest case with the boundary terms as in (2.4) and of three throats in the limit $m \rightarrow \infty$ the BC's will be

$$
\begin{aligned}
& \phi_{1 \mid z_{1}=0}=\phi_{2 \mid z_{2}=0}=\phi_{3 \mid z_{3}=0}, \\
& \partial_{1} \phi_{1 \mid z_{1}=0}+\partial_{2} \phi_{2 \mid z_{2}=0}+\partial_{3} \phi_{3 \mid z_{3}=0}=0 .
\end{aligned}
$$

One can then explicitly calculate that

$$
r=-\frac{1}{3}, \quad \alpha_{2}=\frac{2}{3}, \quad \alpha_{3}=\frac{2}{3},
$$

and thus

$$
|r|^{2}+\left|\alpha_{2}\right|^{2}+\left|\alpha_{3}\right|^{2}=1
$$

This is a simple example of conservation of probability, that is the sum of the reflection coefficient and the transmission coefficients into the different throats adds up to one:

$$
R+\sum_{i=1}^{N} T_{i}=1
$$

One can show that this relation is also satisfied for the general case with the BC's from (2.14). This simple result can be easily generalized to incoming waves on an arbitrary number of throats.

\section{Spectrum of KK modes}

The spectrum of KK modes in the "thin" limit of a multithroat configuration possesses some interesting features. A free-scalar field propagating on $N$ flat throats 
joined at a single UV brane provides a simple case that shows many aspects of the general situation. Applying Neumann BC's at the IR ends of each of the throats $\phi^{\prime}\left(R_{i}^{\prime}\right)=0$ and the simple Kirchhoff-type BC's given in Eqs. (2.6) and (2.7) at the UV brane, the solution in the $i$ 'th throat for the $n$ 'th KK wavefunction is given by

$$
\phi_{i, n}\left(z_{i}\right)=\sqrt{2} C_{n} \frac{\cos \left[q_{n}\left(R_{i}^{\prime}-z_{i}\right)\right]}{\cos \left(q_{n} R_{i}^{\prime}\right)}
$$

where the KK mass-squareds are determined by the solutions for $q_{n}$ of the eigenvalue equation

$$
\sum_{i=1}^{N} \tan \left(q_{n} R_{i}^{\prime}\right)=0 .
$$

The normalization constant $C_{n}$ is found to be

$$
C_{n}=\frac{1}{\sqrt{\sum_{i} R_{i}^{\prime} \sec ^{2}\left(q_{n} R_{i}^{\prime}\right)}} .
$$

If the $N$ throats are symmetric $R_{1}^{\prime}=R_{2}^{\prime}=\ldots R_{N}^{\prime} \equiv R^{\prime}$ then the solutions to the KK momentum eigenvalues are simple

$$
q_{n}=\frac{n \pi}{R^{\prime}}
$$

with normalized wavefunction in the $i$ 'th throat

$$
\phi_{i, n}\left(z_{i}\right)=\sqrt{\frac{2}{N R^{\prime}}} \cos \left[q_{n}\left(R^{\prime}-z_{i}\right)\right] .
$$

Notice that this normalization factor depends upon the total volume of the $N$ throats but the mass gap to the first excited state above the zero mode is $1 / R^{\prime}$. This leads to the possibility of large volume compactifications without the usual associated light KK modes cf. [11].

However if the throats are not symmetric, as we would generically expect, then the spectrum of KK modes can be extremely complicated, even in this very simple flat-throat free-scalar case. In particular, if the throat lengths $R_{i}^{\prime}$ are not all rationally related then the spectrum of KK masses appears to be "chaotic" as can be seen by simple numerical investigation of the eigenvalue Eq. (2.20). Alternatively Dirichlet conditions at the UV brane can lead to the spectrum of $N$ decoupled throats.

\section{FERMIONS IN MULTIPLE THROATS}

Fermions in extra dimensions always require special attention, since chiral fermions appear only after imposing appropriate BC's (see [12] for an extensive discussion of fermion BC's). The reason is that higher dimensional fermions are necessarily nonchiral Dirac fermions. In a single flat extra dimension a 5D fermion is given (in terms of 4D Weyl spinors) as

$$
\left(\begin{array}{l}
\chi(z) \\
\bar{\psi}(z)
\end{array}\right),
$$

where $\chi$ will denote a left-handed 4D Weyl fermion, and $\psi$ a right handed one. The equation of motion in the bulk for such a field with bulk mass $m$ is given by

$$
\begin{aligned}
& -i \bar{\sigma}^{\mu} \partial_{\mu} \chi-\partial_{z_{i}} \bar{\psi}+m \bar{\psi}=0, \\
& -i \sigma^{\mu} \partial_{\mu} \bar{\psi}+\partial_{z_{i}} \chi+m \chi=0 .
\end{aligned}
$$

$\chi, \psi$ satisfy first order differential equations, so one must impose in total two BC's for the system. The simplest BC's are $\left.\psi\right|_{0, R^{\prime}}=0$ (or $\left.\chi\right|_{0, R^{\prime}}=0$ ) which would imply the presence of a chiral zero mode in $\chi$ with wave function $e^{-m z}$ (or for $\psi$ with wave function $e^{m z}$ ). Another possible choice is that of twisted BC's, that is $\left.\psi\right|_{0}=\left.\chi\right|_{R^{\prime}}=0$ (or the other way round) in which case there would be no zero modes.

Here we would like to construct a set-up with throats, which could give a theory with chiral zero modes, that could be localized in either of the throats, or in both. For simplicity we will start with just two throats. If these were not connected at the UV brane, there would be many possibilities for the zero modes. For example, we could have a $\mathrm{LH}$ (or a $\mathrm{RH}$ ) zero mode in each throat, or one $\mathrm{LH}$ in one and one $\mathrm{RH}$ in the other throat (assuming none of the fields are twisted). However, we would like a case, when there is a single (for example LH) zero mode propagating in both throats. This means that we need to start with a separate LH zero mode in both throats, and remove one combination of them. Because of chirality this can only be done by adding a localized RH mode at the UV brane. Consider, for example, the following action $\stackrel{\leftrightarrow}{\partial}_{z_{i}}=\vec{\partial}_{z_{i}}-$ $\left.\overleftarrow{\partial}_{z_{i}}\right)$

$$
\begin{aligned}
S= & \int_{0}^{R_{1}^{\prime}} d^{4} x d z_{1}\left(-i \bar{\chi}_{1} \bar{\sigma}^{\mu} \partial_{\mu} \chi_{1}-i \psi_{1} \sigma^{\mu} \partial_{\mu} \bar{\psi}_{1}\right. \\
& \left.+\left(\frac{1}{2} \psi_{1} \overleftrightarrow{\partial}_{z_{1}} \chi_{1}+m_{1} \psi_{1} \chi_{1}+\text { H.c. }\right)\right) \\
& +\int_{0}^{R_{2}^{\prime}} d^{4} x d z_{2}\left(-i \bar{\chi}_{2} \bar{\sigma}^{\mu} \partial_{\mu} \chi_{2}-i \psi_{2} \sigma^{\mu} \partial_{\mu} \bar{\psi}_{2}\right. \\
& \left.+\left(\frac{1}{2} \psi_{2} \stackrel{\leftrightarrow}{\partial}_{z_{2}} \chi_{2}+m_{2} \psi_{2} \chi_{2}+\text { H.c. }\right)\right) \\
& +\left.\int d^{4} x\left(m^{1 / 2} \psi_{0}\left(\chi_{1}-\chi_{2}\right)+\text { H.c. }\right)\right|_{z=0}
\end{aligned}
$$

Here 1, 2 refer to two throats. We will also assume that the $\mathrm{BC}$ in the absence of the UV brane would have been $\left.\psi_{1}\right|_{0, R_{1}^{\prime}}=\left.\psi_{2}\right|_{0, R_{2}^{\prime}}=0$. The equation of motion with respect to the localized field will then enforce a modified BC

$$
\left.\chi_{1}\right|_{z=0}=\left.\chi_{2}\right|_{z=0},
$$

while integrating the bulk equations of motion for the $\chi_{1,2}$ fields around the UV brane ( $\epsilon$ is a small distance away from the UV brane) imply 


$$
\begin{aligned}
& \bar{\psi}_{1 \mid \epsilon}-\bar{\psi}_{1 \mid 0}-m^{1 / 2} \psi_{0}=0 \\
& \bar{\psi}_{2 \mid \epsilon}-\bar{\psi}_{2 \mid 0}+m^{1 / 2} \psi_{0}=0 .
\end{aligned}
$$

Using the original $\mathrm{BC}$ at $z=0$, we are left with

$$
\left.\psi_{1}\right|_{z=\epsilon}=-\left.\psi_{2}\right|_{z=\epsilon} .
$$

These new BC's will remove one of the combination of zero modes, and the remaining single left-handed zero mode will have the wave function

$$
\chi_{1}(z)=A e^{-m_{1} z}, \quad \chi_{2}(z)=A e^{-m_{2} z},
$$

and the remaining overall coefficient $A$ is determined by the normalization of the kinetic term of this zero mode. If $m_{1}=m_{2}<0$ then the zero mode will be localized at the two IR ends of the two throats. For $m_{1}=m_{2}>0$ the zero mode will be localized around the UV brane in both throats, while for $m_{1} m_{2}<0$ it will be localized at the end of the throat with positive $m$. This is illustrated in Fig. 4.

We can easily generalize the above procedure for the case with arbitrary number $N$ throats. We will again be interested in the case of a single chiral fermion (for example a left-handed one) propagating in all throats. Thus we need to start with a system that has a LH zero mode in all throats (that is with BC's $\left.\psi_{i}\right|_{0, R_{i}^{\prime}}=0$ ). In order to remain with just one overall zero mode we need to remove the additional zero modes via localized mass terms. One possibility would be to add Majorana masses, but we are assuming that there are some conserved $U(1)$ charges that generically forbid Majorana mass terms. Thus we introduce $N-1$ localized RH modes $\xi_{i}$ at the UV brane, and add the localized Lagrangian

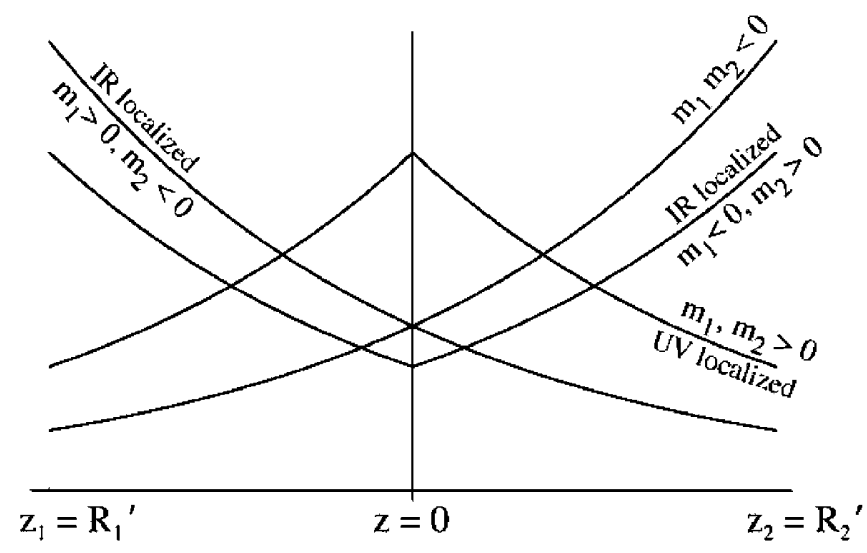

FIG. 4. The wave function of the fermions for various choices of the signs of the bulk masses. For the continuous cases $m_{1} m_{2}<0$, while for the case localized at the UV brane $m_{1}$, $m_{2}>0$. The final case (with localization at the two ends) corresponds to $m_{1}, m_{2}<0$. For all cases we have chosen $\left|m_{1}\right|=$ $\left|m_{2}\right|=1 / R_{1}^{\prime}=1 / R_{2}^{\prime}$.

$$
\int d^{4} x \sum_{i=1}^{N-1} \sum_{j=1}^{N}\left(m^{1 / 2} \tilde{\xi}_{i} \mathcal{K}_{i j} \chi_{j}+\text { H.c. }\right)_{\mid z=0} \text {. }
$$

We will assume that the rank of the $N \times(N-1)$ matrix $\mathcal{K}$ is $N-1$, so that there is not any combination of the localized fields that can be decoupled, and only one zero mode is left in the spectrum. The equations of motion of the localized fields, for the zero mode, will impose the following $N-1$ BC's at $z_{i}=0$ :

$$
\sum_{j} \mathcal{K}_{i j} \chi_{j}=0
$$

The wave functions of the zero mode in the throats is

$$
\chi_{j}=A_{j} e^{-m_{j} z_{j}},
$$

where the coefficients $A_{j}$ are determined, up to an overall normalization, by the BC's on the UV brane. Thus, the matrix $\mathcal{K}$ will determine in which throats the zero mode prefers to live, while, as in the 2-brane case, the signs and magnitudes of the bulk masses can yield to a wide variety of different localization patterns.

A simplified case, similar to the $N$ vibrating rods for a scalar field, is given by the following mass term:

$$
\left.\int d^{4} x \sum_{i=1}^{N-1}\left(m^{1 / 2} \xi_{i}\left(\chi_{i}-\chi_{i+1}\right)+\text { H.c. }\right)\right|_{z=0} .
$$

This will then imply the BC's $\chi_{1}=\chi_{2}=\ldots \chi_{N}$ at $z=0$, and the zero mode will be of the form

$$
\chi_{i}=A e^{-m_{i} z_{i}}, \quad A=\left[\sum_{i=1}^{N}\left(\frac{1-e^{-2 m_{i} R_{i}^{\prime}}}{2 m_{i}}\right)\right]^{-(1 / 2)} .
$$

\section{GAUGE FIELDS}

We now would like to extend the previous multithroat set-up with scalars to allow gauge fields to also propagate in the throats. The IR BC's can be derived exactly as one would do for a single throat theory so we only have to discuss the $\mathrm{BC}$ at the UV brane. The bulk Lagrangians we consider are in flat space:

$$
\mathcal{L}_{\text {bulk }, i}=-\frac{1}{4 g_{5, i}^{2}} F_{(i) M N}^{a 2}
$$

The results for the warped case are briefly summarized at the end of this section.

To find the BC's on the UV brane for the case where a single gauge field propagates in all the throats let us start with $N S U(M)$ gauge fields propagating in $N$ throats and consider how to describe in field theory the UV breaking of the $N$ gauge groups to the diagonal $S U(M)$ gauge group. This can be achieved by introducing $N-1$ bi-fundamental Higgs fields localized at the UV brane at $z_{i}=0$ : 


$$
\mathcal{L}_{\mathrm{UV}}=\sum_{\alpha=1}^{N-1}\left(D_{\mu} \phi_{\alpha}^{\dagger} D^{\mu} \phi_{\alpha}-\lambda\left(\operatorname{Tr} \phi_{\alpha}^{\dagger} \phi_{\alpha}-c_{\alpha}^{2}\right)^{2}\right)
$$

where $\phi_{\alpha}$ transforms as a fundamental of $S U(M)_{\alpha}$ and an antifundamental of $S U(M)_{\alpha+1}$. The gauge covariant derivative is given by ( $T^{a}$ are the generators of the gauge group in the fundamental representation and $A_{(\alpha) \mu}^{a}$ are the gauge fields in $\alpha$ th throat)

$$
D_{\mu} \phi_{\alpha}=\partial_{\mu} \phi_{\alpha}-i\left(A_{(\alpha) \mu}^{a} T^{a} \phi_{\alpha}-\phi_{\alpha} T^{a} A_{(\alpha+1) \mu}^{a}\right)
$$

As usual we expand the Higgs around its VEV:

$$
\phi_{\alpha}=\frac{e^{i \pi_{\alpha} / f}}{\sqrt{2}}\left(v+h_{\alpha}\right) \quad \alpha=1 \ldots N-1,
$$

where $\phi_{\alpha}, v$, and $h_{\alpha}$ are $M \times M$ matrices and $\pi_{\alpha}=\pi_{\alpha}^{a} T^{a}$ and $f^{2}=\operatorname{Tr} v^{\dagger} T^{a} T^{a} v$. Since we are interested in breaking to the diagonal subgroup we will take $v$ to be proportional to the identity. The Lagrangians (4.1) and (4.2) contain some bulk and brane mixing terms involving $A_{\mu}$ that we want to cancel out with a generalized $R_{\xi}$ gauge fixing term. Expanding up to quadratic order:

$$
\begin{aligned}
\mathcal{L}_{\text {eff }}= & \sum_{i=1}^{N} \int_{0}^{R_{i}^{\prime}} d z_{i}\left\{\frac{1}{g_{5, i}^{2}}\left(-\frac{1}{4} F_{(i) \mu \nu}^{a 2}+\frac{1}{2}\left(\partial_{z_{i}} A_{(i) \mu}^{a}\right)^{2}+\frac{1}{2}\left(\partial_{\mu} A_{(i) 5}^{a}\right)^{2}-\partial_{\mu} A_{(i) 5}^{a} \partial_{z_{i}} A_{(i)}^{a \mu}\right)\right\} \\
& +\sum_{\alpha=1}^{N-1} \frac{1}{2} \operatorname{Tr} v^{\dagger}\left(\frac{1}{f} \partial^{\mu} \pi_{\alpha}-A_{(\alpha)}^{\mu}+A_{(\alpha+1)}^{\mu}\right)\left(\frac{1}{f} \partial_{\mu} \pi_{\alpha}-A_{(\alpha) \mu}+A_{(\alpha+1) \mu}\right) v \\
& +\sum_{\alpha=1}^{N-1} \frac{1}{2} \operatorname{Tr} \partial_{\mu} h_{\alpha}^{\dagger} \partial^{\mu} h_{\alpha}-\lambda\left(\operatorname{Tr} v^{\dagger} h_{\alpha}\right)^{2}+\ldots
\end{aligned}
$$

Integrating the derivative mixing term by parts gives:

$$
\int_{0}^{R_{i}^{\prime}} d z_{i} A_{(i) 5}^{a} \partial_{z_{i}} \partial_{\mu} A_{(i)}^{a \mu}=-\int_{0}^{R_{i}^{\prime}} d z_{i} \partial_{z_{i}} A_{(i) 5}^{a} \partial_{\mu} A_{(i)}^{a \mu}+\left[A_{(i) 5}^{a} \partial_{\mu} A_{(i)}^{a \mu}\right]_{0}^{R_{i}^{\prime}} .
$$

The mixing terms between vectors and scalars are then cancelled by adding the bulk and the brane gauge fixing Lagrangians (for simplicity we will take all the brane gauge fixing parameters to be equal to $\xi_{b}$ ):

$$
\begin{aligned}
\mathcal{L}_{\mathrm{GF}}= & -\sum_{i=1}^{N} \frac{1}{g_{5, i}^{2}} \frac{1}{2 \xi_{i}} \int d z_{i}\left(\partial_{\mu} A_{(i)}^{a \mu}-\xi_{i} \partial_{z_{i}} A_{(i) 5}^{a}\right)^{2}-\left.\sum_{i=1}^{N} \frac{1}{2 \xi_{b}}\left(\partial_{\mu} A_{(i)}^{a \mu}+\xi_{b}\left(f\left(\pi_{i}^{a}-\pi_{i-1}^{a}\right)-\frac{A_{(i) 5}^{a}}{g_{5, i}^{2}}\right)\right)^{2}\right|_{z_{i}=0} \\
& -\left.\sum_{i=1}^{N} \frac{1}{2 \xi_{b}}\left(\partial_{\mu} A_{(i)}^{a \mu}+\xi_{b} \frac{A_{(i) 5}^{a}}{g_{5, i}^{2}}\right)^{2}\right|_{z_{i}=R_{i}^{\prime}}
\end{aligned}
$$

(with the convention that $\pi_{0}^{a}=0$ and $\pi_{N}^{a}=0$ ). The unitary gauge is realized in the limit where all the $\xi$ 's are sent to infinity. The full Lagrangian then leads to the following equation of motion for $A_{\mu}\left(q_{i}\right.$ denotes the $4 \mathrm{D}$ momentum):

$$
\partial_{z_{i}}\left(\partial_{z_{i}} A_{(i) \mu}\right)+\left(q^{2} \eta_{\mu \nu}+\left(\frac{1}{\xi_{i}}-1\right) q_{\mu} q_{\nu}\right) A_{(i)}^{\nu}=0,
$$

while the BC's, fixed by requiring the vanishing of the boundary variation terms in Eq. (4.5), are:

$$
\partial_{z_{i}} A_{(i) \mu}^{a}-g_{5, i}^{2} f^{2}\left(A_{(i) \mu}^{a}-A_{(i+1) \mu}^{a}\right)=0 .
$$

In the infinite VEV limit, the corresponding UV BC's are

$$
\begin{gathered}
A_{(i) \mu}^{a}=A_{(i+1) \mu}^{a} \\
\frac{\partial_{z_{1}} A_{(1) \mu}^{a}}{g_{5,1}^{2}}+\ldots+\frac{\partial_{z_{N}} A_{(N) \mu}^{a}}{g_{5, N}^{2}}=0
\end{gathered}
$$

These BC's are analogous to case of scalar fields propagat- ing in multiple throats, thus the KK expansion of these fields will have identical properties for the two cases.

Next we would like to discuss the question of under what circumstances will there be physical modes in the scalar components. The bulk equation of motion for the scalar fields $A_{(i) 5}$ is:

$$
\xi_{i} \partial_{z}^{2} A_{(i) 5}^{a}+q^{2} A_{(i) 5}^{a}=0
$$

From this we see that the only physical modes that can survive in the unitary gauge limit $\xi \rightarrow \infty$ are those with $q^{2}=0$. Thus for theses modes we have

$$
\partial_{z}^{2} A_{(i) 5}^{a}=0
$$

The BC's for the $\pi$ 's and $A_{(i) 5}$ 's are obtained by requiring that the variation of the action vanishes at the UV brane: 


$$
\begin{aligned}
\delta \mathcal{L}_{\text {scalar }}= & -\sum_{\alpha=1}^{N-1} \partial_{\mu} \partial^{\mu} \pi_{\alpha}^{a} \delta \pi_{\alpha}^{a} \\
& +\left.\sum_{i=1}^{N} \frac{\xi_{i}}{2 g_{5, i}^{2}} \delta A_{(i) 5}^{a} \partial_{z_{i}} A_{(i) 5}^{a}\right|_{z_{i}=0} \\
- & \left.\sum_{i=1}^{N} \xi_{b}\left(f\left(\pi_{i}^{a}-\pi_{i-1}^{a}\right)-\frac{A_{(i) 5}^{a}}{g_{5, i}^{2}}\right)\right|_{z_{i}=0} \\
& \times\left(f\left(\delta \pi_{i}^{a}-\delta \pi_{i-1}^{a}\right)-\frac{\delta A_{(i) 5}^{a}}{g_{5, i}^{2}}\right)
\end{aligned}
$$

In the unitary gauge the terms in the $A_{5}$ and $\pi$ equations of motion that are multiplied by gauge parameters must vanish. So we see that $\pi_{1}^{a}$ and $\pi_{N-1}^{a}$ are fixed in terms of $A_{(1) 5}^{a}$ and $A_{(N) 5}^{a}$. The remaining $N-2$ equations from $\xi_{b}$ terms fix the remaining $\pi_{i}^{a}$ 's and require

$$
0=\left.\sum_{i=1}^{N} \frac{A_{(i) 5}^{a}}{g_{5, i}^{2}}\right|_{z_{i}=0}
$$

By requiring that the terms proportional to $\xi_{i}$ vanish, we also obtain

$$
\left.\partial_{z_{i}} A_{(i) 5}^{a}\right|_{z_{i}=0}=0
$$

We can see that as expected, the BC's for the $A_{5}$ components are the same as for the $A_{\mu}$ components, except that the Dirichlet $\mathrm{BC}$ is replaced by a Neumann $\mathrm{BC}$ and vice versa. The BC's (4.16) and (4.17) together with the bulk equation of motion implies that for any physical mode the $A_{(i) 5}$ 's have to be constant in every throat, and due to the BC (4.16) these constants have to add up to zero. Thus there can at most be $N-1$ independent scalar modes. How many of these $N-1$ are actually physical will depend on the BC's at the IR branes. If there is no additional symmetry breaking on any brane, or if there is symmetry breaking on at most one brane, then none of the scalar $A_{(i) 5}$ modes will be physical. The reason is that the $\mathrm{BC}$ on a given IR brane for $A_{(i) 5}$ will be Neumann $\left.\partial_{z_{i}} A_{(i) 5}^{a}\right|_{z_{i}=R^{\prime}}=0$ for the case when a particular generator $a$ is broken by a VEV of a scalar field on the brane, while it will be Dirichlet $\left.A_{(i) 5}^{a}\right|_{z_{i}=R^{\prime}}=0$ for the case there is no symmetry breaking. If there is a Dirichlet $\mathrm{BC}$ at the bottom of at least $N-1$ throats then at least $N-1$ of the $A_{(i) 5}$ 's are set to zero, and since all $N$ have to also add up to zero, we see that they all have to vanish. So there is no physical mode left. Thus in order for a physical scalar zero mode to exist in any particular gauge direction, the generator corresponding to that direction has to be broken at least twice (on the bottom of at least two throats). This also immediately implies, that if a particular generator is broken on $k$ throats, there will be $k-1$ physical scalar zero modes appearing.

The counting of the physical scalar modes has a very simple holographic interpretation (at least for the case when all the throats are warped). If the extra dimensions were warped, then the set-up we have produced would correspond to $N$ different 4D CFT's (corresponding to the bulks of the warped throats), each of which has an $S U(M)$ global symmetry. The set-up around the UV brane ensures that only the diagonal subgroup of these global symmetries is actually gauged. Then if the symmetry is broken on the bottom of $k$ throats that will correspond to $k$ of the CFT's spontaneously breaking its own global symmetry, each of which will produce a Goldstone boson. However, since the diagonal subgroup is gauged, one combination of these Goldstone bosons is eaten, leaving us with $k-1$ physical massless Goldstone modes. We have seen that the BC's derived here exactly reproduce this expected answer. Even though we have only considered the flat-throat case here, the generalization to the warped case is straightforward. With bulk Lagrangians of the form

$$
\mathcal{L}_{\text {bulk }, i}=-\mathcal{K}_{(i)}\left(z_{i}\right) \frac{1}{4 g_{5, i}^{2}} F_{(i) M N}^{a 2},
$$

following the same procedure as before, we obtain the following $\mathrm{BC}$ 's for the $A_{\mu}$ components in the infinite VEV limit

$$
\begin{gathered}
A_{(i) \mu}^{a}=A_{(i+1) \mu}^{a} \\
\frac{\mathcal{K}_{(1)}(z)}{g_{5,1}^{2}} \partial_{z_{1}} A_{(1) \mu}^{a}+\ldots+\frac{\mathcal{K}_{(N)}(z)}{g_{5, N}^{2}} \partial_{z_{N}} A_{(N) \mu}^{a}=0
\end{gathered}
$$

while the $\mathrm{BC}$ 's for the $A_{5}$ components read

$$
\begin{aligned}
0 & =\left.\sum_{i=1}^{N} \frac{\mathcal{K}_{(i)}}{g_{5, i}^{2}} A_{(i) 5}^{a}\right|_{z_{i}=R} \\
0 & =\left.\partial_{z_{i}}\left(\mathcal{K}_{(i)} A_{(i) 5}^{a}\right)\right|_{z_{i}=R}
\end{aligned}
$$

\section{GRAVITY}

Let us finally discuss how an appropriate gravitational background and fluctuations could be obtained in these throats. For useful references for this section see [13]. For the background metric we will be assuming that it is conformally flat in every throat and is given by

$$
g_{M N}^{(i)}=e^{-A_{i}\left(z_{i}\right)}\left(d x^{\mu} d x^{\nu} \eta_{\mu \nu}-d z_{i}^{2}\right),
$$

where the coordinates $z_{i}$ run from the UV brane $z_{i}=0$ to the individual IR branes $z_{i}=R_{i}^{\prime}$. We will assume that the metric is continuous at the UV brane, that is

$$
A_{i}=A_{i+1}=0 \text {. }
$$

Here we have rescaled the overall warp factor on the UV brane to be equal to one. If there are different cosmological constants $\Lambda_{i}$ in the different throats, then the warp factors will be given by 


$$
A_{i}\left(z_{i}\right)=2 \log \left(z_{i} / R_{i}+1\right)
$$

where the AdS curvatures in the different throats are given by their usual expressions

$$
1 / R_{i}^{2}=-\frac{\kappa^{2} \Lambda_{i}}{6}
$$

and $\kappa^{2}$ is the 5D Newton constant (related to the 5D Planck scale by $\left.\kappa^{2}=1 /\left(2 M_{*}^{3}\right)\right)$. These will completely fix the solutions, however one still has to satisfy the generalized Israel junction condition [14]. In this case it will be given by

$$
\sum_{i} \partial_{z_{i}} A_{i \mid z_{i}=0}=\frac{2 \kappa^{2}}{3} T
$$

where $T$ is the tension of the junction (i.e. the UV brane). This will just result in the usual Randall-Sundrum (RS)type fine tuning between the brane tensions and the various throat cosmological constants:

$$
\sum_{i} \frac{1}{R_{i}}=\frac{\kappa^{2}}{3} T
$$

One can also find the graviton KK modes easily in this background. Assuming that the metric fluctuations are of the form

$$
d s_{i}^{2}=e^{-A_{i}\left(z_{i}\right)}\left(d x^{\mu} d x^{\nu}\left(\eta_{\mu \nu}+h_{\mu \nu}^{(i)}\left(x, z_{i}\right)\right)-d z_{i}^{2}\right)
$$

and using transverse traceless gauge (that is neglecting the radion-type scalar solutions) $h_{\nu}^{\mu}=\partial^{\mu} h_{\mu \nu}=0$ we find that the Einstein equations in the throats give the usual RS-type equations:

$$
\left(\partial^{\mu} \partial_{\mu}-\partial_{z_{i}}^{2}\right) h_{\mu \nu}^{(i)}+\frac{3}{2} \partial_{z_{i}} A_{i} \partial_{z_{i}} h_{\mu \nu}^{(i)}=0 .
$$

All the terms including the brane tension are compensated by the effects of the background metric, so the generalized Israel junction condition at the UV brane for the perturbations will just be:

$$
\sum_{i} \partial_{z_{i}} h_{\mu \nu}^{(i)}=0,
$$

while the metric should still be continuous on the UV brane requiring

$$
h_{\mu \nu}^{(i)}=h_{\mu \nu}^{(i+1)} .
$$

Thus the BC's for the graviton modes are completely analogous to the case of scalars and gauge bosons with a single zero mode. For the particular case of a graviton zero mode in a warped throat (à la RS), we can easily solve these equations, since the solution of (5.8) are just constant in every throat, with the constants equal at the UV brane. The overall normalization would then be fixed by calculating the kinetic term of the graviton zero mode.

\section{APPLICATIONS}

\section{A. A throat for every family}

One obvious application of the multithroat construction is to have a set-up where the gauge bosons propagate on all the throats but a family of quarks and leptons lives in a single throat. With three throats, we can have the three families each propagating in different throats. In order to be able to generate mixings among different families the Higgs field has to have an overlap with the UV brane. Thus one would expect that in order to solve the gauge hierarchy problem one would have to make the set up supersymmetric. The supersymmetric flavor problem could then be resolved geometrically, if one assumes that the three throats were identical (that is they are related by an $S_{3}$ exchange symmetry). In this case one could imagine that the only source of supersymmetry breaking was due to strong dynamics at the bottom of the throats giving rise to equal soft supersymmetry breaking terms localized on the IR branes. Since we are assuming that the three throats are identical, one will get equal soft breaking terms for the three families, and since they are separated in different throats they will be flavor diagonal. In order for the Higgs to pick up soft breaking terms it has to then propagate in the three throats. Since we are assuming that the three throats are identical this will also imply that the Yukawa couplings in the throats are equal for the three families. A hierarchy among the three families can then be obtained by adding terms to the UV brane such that the VEV's of the Higgses in the three different throats will be the largest for the throat containing the third generation, and the smallest for the first generation. The mixings among the generations and the differences of masses within a generation can then be adjusted by adding some additional UV-brane localized Yukawa couplings. The set up is illustrated in Fig. 5.

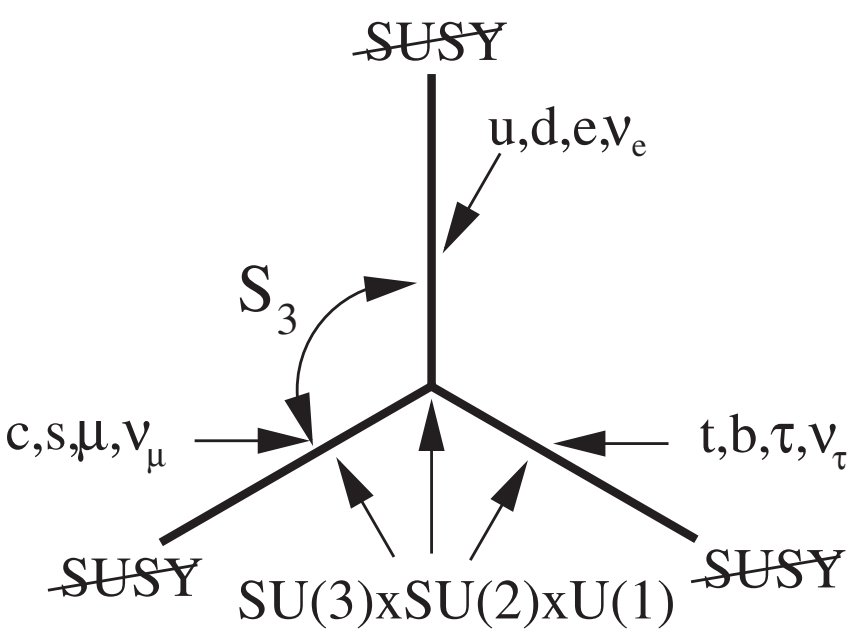

FIG. 5. Illustration of the throat construction of the model with a throat for every family. 


\section{B. A GUT model for doublet-triplet splitting}

One can embed this scenario in a model of doublettriplet splitting in SUSY GUTs along the lines of $[15,16]$. Thus these GUT models can be given a simple geometric interpretation. The essence of the model in [15] is to have three $S U(5)$ groups broken by various bifundamental fields $Z_{i}$ and $\bar{Z}_{i}$ to the diagonal $S U(3) \times S U(2) \times U(1)$ subgroup. A key element is the $S_{3}$ exchange symmetry of the three $S U(5)$ factors and an additional $Z_{2}$ symmetry that will forbid direct mass terms of the Higgs fields.

The geometric construction is again based on a supersymmetric three-throat set up, each of which have an $S U(5)$ group propagating in them. The three throats have again an $S_{3}$ exchange symmetry as in Fig. 6. We assume that half of the eight supercharges are broken on the IR brane via BC's, and thus only an $\mathcal{N}=1$ set of fields have light modes. On the UV brane we introduce the bifundamental fields $Z_{i}, \bar{Z}_{i}$ and a generic superpotential (as in [15])

$$
W(Z)_{\mathrm{UV}}=M_{1} \sum_{j}\left(Z_{j} \bar{Z}_{j}\right)+\frac{1}{M_{2}} \sum_{j \neq k}\left(Z_{j} \bar{Z}_{j}\right)\left(Z_{k} \bar{Z}_{k}\right)+\ldots,
$$

where the extra terms correspond to additional dimension four superpotential terms. Among the possible set of supersymmetric vacua are those with VEVs

$$
\begin{gathered}
\left\langle Z_{1}\right\rangle=\left\langle\bar{Z}_{1}\right\rangle=v_{1} \operatorname{diag}(1,1,1,0,0), \\
\left\langle Z_{2}\right\rangle=\left\langle\bar{Z}_{2}\right\rangle=\left\langle Z_{3}\right\rangle=\left\langle\bar{Z}_{3}\right\rangle=v_{2} \operatorname{diag}(1,1,1,1,1) .
\end{gathered}
$$

With these VEVs the three $S U(5)$ 's in the three throats are broken to just the SM groups. There are six separate Higgs fields $H_{i}, \bar{H}_{i}$ (which are originally six hypermultiplets, but due to the BC's on the IR brane only one chiral component will have a zero mode). In order to forbid the direct mass term for these fields one needs to assume that there is a $Z_{2}$ symmetry $\{\bar{H}, Z, \bar{Z}\} \rightarrow-\{\bar{H}, Z, \bar{Z}\}$ which will only allow the superpotential coupling on the UV brane

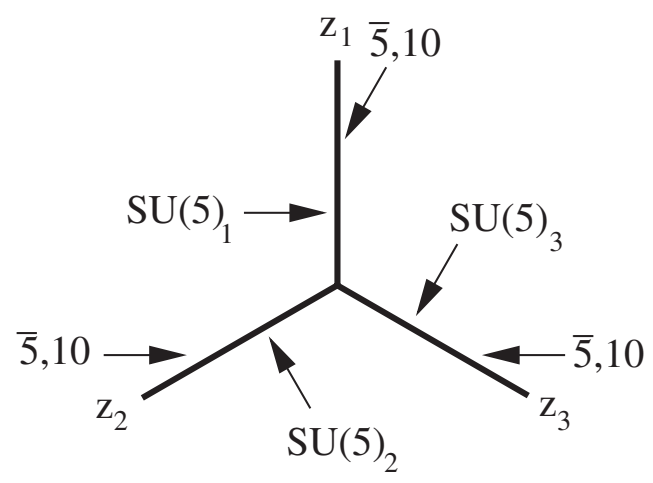

FIG. 6. Illustration of the throat construction of the $S U(5)^{3}$ model.

$$
W(H, Z)_{\text {trilinear }}=\lambda \sum_{i, k} H_{i} Z_{i k} \bar{H}_{k} \text {, }
$$

where we have defined $Z_{i k}=Z_{j}$ for $j \neq i$, $k$. With this additional UV-brane superpotential all components of the Higgs fields $H_{i}, \bar{H}_{i}$ will get a mass of order $v_{1,2}$, except for one pair of $S U(2)$ doublets, which will be identified with the MSSM Higgs doublets. This will also automatically solve the $\mu$-problem, and an order $M_{\text {weak }} \mu$-term can be generated if after minimizing the potential for the $Z$, $\bar{Z}$-fields the VEV's will get shifted due to the addition of the soft breaking terms. The model of [16] can be implemented similarly, with the added convenience that the UVbrane VEV's of the $Z, \bar{Z}$ can now be interpreted as Wilson lines along the coordinates parameterizing the direction along the UV head.

\section{A simple geometric construction for trinification}

Quite a lot of effort has been devoted [17] to naturally implement a grand unified trinification [18] model based on the gauge group $S U(3)^{3} / Z_{3}$ into extra dimensions. The multithroat construction clearly offers a simple implementation for such a theory. In 4D the gauge group is given by $S U(3)_{c} \times S U(3)_{L} \times S U(3)_{R}$, with $S U(3)_{c}$ being ordinary $\mathrm{QCD}, S U(2)_{L} \subset S U(3)_{L}$, and $Y=-\frac{1}{\sqrt{3}}\left(T_{L}^{8}+\sqrt{3} T_{R}^{3}+\right.$ $\left.T_{R}^{8}\right)$. One family of SM matter can be embedded into the representation $27=(3, \overline{3}, 1)+(1,3, \overline{3})+(\overline{3}, 1,3)$. This contains the usual SM fermions, plus some additional massive vectorlike fields: an additional $S U(2)_{L}$ singlet vectorlike bottom, an additional vectorlike $S U(2)_{L}$ doublet (color singlet) plus two right handed neutrinos.

The symmetry breaking is obtained by at least two Higgs fields in the same representation 27. Clearly, since QCD is unbroken only the $(1,3, \overline{3})$ component of these higgses can get a VEV, and the assumption is that one of these higgses $\Phi$ will get a VEV of the form

$$
\langle\Phi\rangle=\left(\begin{array}{l}
\quad \\
\quad
\end{array}\right)
$$

breaking $S U(3)_{L} \times S U(3)_{R}$ to $S U(2)_{L} \times S U(2)_{R} \times$ $U(1)_{B-L}$, while the other Higgs $\chi$ has a VEV of the form

$$
\langle\chi\rangle=\left(v^{\prime}\right)
$$

breaking the group to a different $3-2-2-1$ subgroup. In total the two VEV's would leave $3-2-1$ invariant. Electroweak symmetry breaking is achieved if further (electrically and color neutral) components of the Higgs fields get VEV's of the order of the electroweak scale.

The extra dimensional construction based on throats would work as follows: fermions are bi-fundamentals under the $S U(3)$ groups, and assuming they live in separate throats, the individual $S U(3)$ gauge bosons have to be propagating in two separate throats each: 


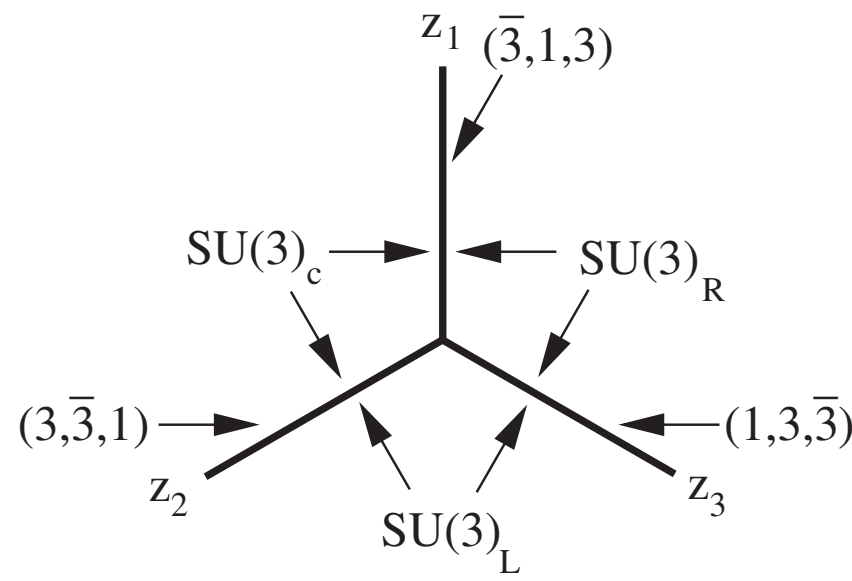

FIG. 7. Illustration of the throat construction of the model for trinification.

$$
\begin{gathered}
S U(3)_{c} \rightarrow\left(z_{1}, z_{2}\right), \quad S U(3)_{L} \rightarrow\left(z_{2}, z_{3}\right), \\
S U(3)_{R} \rightarrow\left(z_{1}, z_{3}\right) .
\end{gathered}
$$

This can be achieved using a construction for the gauge fields of the sort presented in Sec. IV. Fermions are then localized in single throats, obviously with the above assignment of the gauge groups we would find

$$
(3, \overline{3}, 1) \rightarrow z_{2}, \quad(1,3, \overline{3}) \rightarrow z_{3}, \quad(\overline{3}, 1,3) \rightarrow z_{1}
$$

This is illustrated in Fig. 7. Since the symmetry breaking should be felt by all the fermions, the Higgs fields should be localized on the Planck brane. Thus in order to explain the hierarchy the theory should be supersymmetric. Fermion hierarchies could then be explained by varying the localization parameters of the fermions.

\section{CONCLUSIONS}

We have shown how a general theory on a manifold with many throats can be described in a field theory limit as a collection of five dimensional warped spaces intersecting on a single UV brane. We have also shown how the Lagrangian on the UV brane determines the BC's for the extra dimensional fields, giving examples with scalars, gauge bosons, and fermions. This set-up provides a whole new set of tools for model builders to exploit. We have shown the appropriate BC's for scalar, fermion, gauge boson and graviton fields. We have provided three very simple sample applications of these ideas. In the first the three generations of the MSSM propagate in separate throats, and the supersymmetric flavor problem is solved via the exchange symmetry of the three throats. The second example we show how this scenario can be embedded in a beautiful model of doublet-triplet splitting in SUSY GUTs which can be also interpreted geometrically. The third example provides a geometric implementation of trinification.

\section{ACKNOWLEDGMENTS}

We thank Nemanja Kaloper and John March-Russel for collaborations at the early stage of this project. We thank Hsin-Chia Cheng, Jack Gunion, Ryuichiro Kitano, Yasunori Nomura, and Michael Peskin for useful discussions and comments. The research of G. C. and C. C. is supported in part by the DOE OJI grant No. DE-FG02$01 E R 41206$ and in part by the NSF grants No. PHY0139738 and PHY-0098631. C. G. is supported in part by the RTN European Program MRTN-CT-2004-503369 and by the ACI Jeunes Chercheurs 2068. J. T. is supported by the US Department of Energy under grant No. DE-FG0291ER40674. This collaboration is partly supported by the CNRS/USA grant No. 3503. We also thank the Aspen Center for Physics for its hospitality while part of this project has been performed.
[1] N. Arkani-Hamed, S. Dimopoulos, and G. R. Dvali, Phys. Lett. B 429, 263 (1998).

[2] L. Randall and R. Sundrum, Phys. Rev. Lett. 83, 3370 (1999).

[3] Y. Kawamura, Prog. Theor. Phys. 105, 999 (2001); G. Altarelli and F. Feruglio, Phys. Lett. B 511, 257 (2001); L. J. Hall and Y. Nomura, Phys. Rev. D 64, 055003 (2001).

[4] G. Servant and T.M.P. Tait, Nucl. Phys. B650, 391 (2003); H. C. Cheng, J.L. Feng, and K. T. Matchev, Phys. Rev. Lett. 89, 211301 (2002); K. Agashe and G. Servant, Phys. Rev. Lett. 93, 231805 (2004).
[5] N. S. Manton, Nucl. Phys. B158, 141 (1979); I. Antoniadis, K. Benakli, and M. Quiros, New J. Phys. 3, 20 (2001); C. Csáki, C. Grojean, and H. Murayama, Phys. Rev. D 67, 085012 (2003); C. A. Scrucca, M. Serone, and L. Silvestrini, Nucl. Phys. B669, 128 (2003).

[6] C. Csáki, C. Grojean, H. Murayama, L. Pilo, and J. Terning, Phys. Rev. D 69, 055006 (2004).

[7] C. Csáki, C. Grojean, L. Pilo, and J. Terning, Phys. Rev. Lett. 92, 101802 (2004).

[8] H. L. Verlinde, Nucl. Phys. B580, 264 (2000); I. R. Klebanov and M. J. Strassler, J. High Energy Phys. 08 (2000) 052; S. B. Giddings, S. Kachru, and J. Polchinski, 
Phys. Rev. D 66, 106006 (2002); S. Kachru, R. Kallosh, A. Linde, and S.P. Trivedi, Phys. Rev. D 68, 046005 (2003).

[9] S. Dimopoulos, S. Kachru, N. Kaloper, A. E. Lawrence, and E. Silverstein, Phys. Rev. D 64, 121702 (2001); Int. J. Mod. Phys. A 19, 2657 (2004); N. Barnaby, C. P. Burgess, and J. M. Cline, J. Cosmol. Astropart. Phys. 04 (2005) 007.

[10] G. Cacciapaglia, C. Csáki, C. Grojean, M. Reece, and J. Terning, Phys. Rev. D 72, 095018 (2005).

[11] N. Kaloper, J. March-Russell, G. D. Starkman, and M. Trodden, Phys. Rev. Lett. 85, 928 (2000).

[12] C. Csáki, C. Grojean, J. Hubisz, Y. Shirman, and J. Terning, Phys. Rev. D 70, 015012 (2004).

[13] N. Arkani-Hamed, S. Dimopoulos, G. R. Dvali, and N. Kaloper, Phys. Rev. Lett. 84, 586 (2000); C. Csáki and Y. Shirman, Phys. Rev. D 61, 024008 (2000); C. Csáki, J. Erlich, T.J. Hollowood, and Y. Shirman,
Nucl. Phys. B581, 309 (2000).

[14] W. Israel, Nuovo Cimento B 44, 1 (1966).

[15] R. Barbieri, G. R. Dvali, and A. Strumia, Phys. Lett. B 333, 79 (1994); Nucl. Phys. B435, 102 (1995); C-L. Chou, Phys. Rev. D 58, 093018 (1998).

[16] E. Witten, hep-ph/0201018.

[17] C. D. Carone and J. M. Conroy, Phys. Rev. D 70, 075013 (2004); A. Demaria and R. R. Volkas, Phys. Rev. D 71, 105011 (2005); C. D. Carone, Phys. Rev. D 71, 075013 (2005); J.E. Kim, Phys. Lett. B 591, 119 (2004); K. S. Choi and J.E. Kim, Phys. Lett. B 567, 87 (2003); I. Gogoladze, Y. Mimura, and S. Nandi, Phys. Rev. D 69, 075006 (2004).

[18] S. Glashow, in Workshop on Grand Unification, Providence, 1984, edited by K. Kang et al. (World Scientific, Singapore, 1984). 